Journal of Engineering and Science Research 3 (2): 14-20, 2019

e-ISSN: 2289-7127

(C) RMP Publications, 2019

DOI: $10.26666 / \mathrm{rmp}$.jesr.2019.2.3

\title{
Integrator-Backstepping Based Control for Nonlinear Roll-to-Roll Web Dynamics
}

\author{
Vi H. Nguyen " and Thanh T. Tran \\ Faculty of Engineering, Vietnamese-German University, \\ 02 Le Lai, Thu Dau Mot City, Binh Duong Province, Vietnam.
}

\begin{abstract}
The paper presents a method for control analysis and design of roll-to-roll (R2R) dynamic system for printed electronics technology. The research provides an advanced control analysis and design methodology for a general multioutput multi-input (MIMO) strict-feedback class of nonlinear dynamic systems. The research starts with an integrator-backstepping control (IBSC) law formulation for the system by defining the modified tracking error in term of integral of normal tracking error. Considering each sublayer of control design process via a chosen Control Lyapunov function (CLF) and parameter assignment results in anIBSC law. Then, an IBSC law-based control strategy is provided for the design model. For the application of the proposed method, dynamic analysis on roll-to-roll (R2R) web system is made to transform a general nonlinear R2R web dynamics into a standard MIMO strict-feedback form for control analysis and design purposes. With the achieved model, an IBSC-based control algorithm is provided for numerical simulation and experiments. A control software is developed for implementing studies of numerical simulation and experiments to validate the reliability and feasibility of the proposed method
\end{abstract}

\section{Keywords:Integrator-backstepping, nonlinear control, roll-to-roll system}

\section{INTRODUCTION}

Roll-to-roll (R2R) manufacturing technology plays an important role in printed electronics in order to make devices at high speeds with lower cost. With the rapid development of mathematical tools and highperformance computers, investigations on control analysis and design for nonlinear R2R web systems are addressed by many researchers [1][2]. The standard approach for designing controllers for nonlinear dynamic systems is gain-scheduling. In this strategy, linear approximation of dynamic equations at several important operating points is achieved. Depending on these points, linear controllers are designed and then combined continuously from one operating point to another. Due to linearization, the actual system performance and stability can be significantly different from the design results due to the approximated nonlinearities in [1]. In recent years, control analysis and design of nonlinear dynamic systems are addressed by researchers [3]-[10]. A nonlinear observer-based control method is proposed in [3]-[4] in replacement of tension transducers (or loadcells) and estimation of friction and inertia of rewinder and unwinder. Achieving an accurate mathematical model [5]-[8] is one of the most important aspects in control analysis and design for R2R web dynamic system. In these approaches, better assumptions compared to standard models are made to achieve more accurate models. Works in [9]-[14] propose control strategies on disturbance rejection. The works aim to reject these disturbances caused by unknown shapes of rolls. In order to reject the tension disturbance due to the roll shape, an adaptive eccentricity estimator and eccentricity compensation methods are proposed to improve performance [11]. Works in [2,15]-[18], developed by authors of this paper, present backstepping-based control methods to improve the precision and stability. However, the variation of the radius of rewinder and unwinder is not included in these works.

In this paper, an integrator is introduced in the analysis and design process for MIMO strict-feedback model of nonlinear dynamic systems, and then systematic steps are provided for formulating the IBSC law for the model. An IBSCbased control algorithm is proposed for nonlinear two-span R2R web dynamic system with design parameters selected optimally by using the modified genetic algorithm [11]. Numerical simulation studies validate and compare to the work [17] in which a backstepping-based control without an integrator is implemented.

\footnotetext{
*Corresponding Author: Vi Hong Nguyen, Vietnamese-German University, Binh Duong, email: vi.nh@ vgu.edu.vn
} 


\section{EXPMATHEMATICAL BACKGROUND}

In this section, a definition and theorem are provided to assist in developing the proposedcontrol design for a strict-feedback system and a theorem on the convergence of integral-coordinate transformation is provided and proven to support the IBSC method. Then, a systematic procedure is presented for formulating the IBSC law for a MIMO strict-feedback system.

\section{Concepts and Theorem}

Consider a general equation of nonlinear dynamic systemsas

$$
\dot{x}=f(x)
$$

Where $x, f \in \mathrm{R}^{\mathrm{n}}$

Definition 1. (Control Lyapunov Function)

A function $\mathrm{V}(\mathrm{x}): \mathrm{R}^{\mathrm{n}} \rightarrow \mathrm{R}$ is said to be control Lyapunov function (CLF) with respect to the system (1) if the Lie derivative of $\mathrm{V}(\mathrm{x})$ with respect to $\mathrm{f}(\mathrm{x})$ such that

$$
\dot{V}(x)=\frac{\partial V(x)}{\partial x} f(x)=-W(x)<0, \forall x \neq 0
$$

where $\mathrm{W}(\mathrm{x})$ is a positive definitive function.

Theorem 1. (Lyapunov Asymptotic Stability)

The system (1) is said to be asymptotically stable at an equilibrium state in the sense of Lyapunov if there exists a function $\mathrm{V}(\mathrm{x}): \mathrm{R}^{\mathrm{n}}$---- $>\mathrm{R}$ such that $\mathrm{V}(\mathrm{x})$ is a positive definite, decrescent, and CLF.

Consider a coordinate transformation as

$$
\zeta_{1}=\left(x_{1}-x_{r e f}\right)+c_{0} \int_{0}^{t} e(\tau) d \tau
$$

Where $\zeta_{1}$ is a state in new coordinate, $x_{1}$ is a state in original coordinate, $x_{r e f}$ is a constant, $c_{0}$ is a positive gain, $\mathrm{t}$ is a time variable and $e(t)$ is defined as normal tracking error.

\section{Theorem 2: (Integral Convergence)}

If the state $\zeta_{1}$ in equation (3) tends to a zero value, then the corresponding state $x_{1}$ will converge to the command value $x_{r e f}$ for all positive gains $\mathrm{c} 0$ as $\mathrm{t}$ goes to the infinity. Proof Assume the state $\zeta_{1}$ tends to a zero value at a certain time t. Then, the equation (3) can be rewritten as

$$
\left(x_{1}-x_{\text {ref }}\right)+c_{0} \int_{0}^{t} e(\tau) d \tau=0(4)
$$

By using the expression $e(t)=\left(x_{1}-x_{r e f}\right.$, equation (4) can be re-written as

$$
e(t)+c_{0} \int_{0}^{t} e(\tau) d \tau=0(5)
$$

Thus, taking the derivative in time on both sides of the equation (5) yields

$$
\dot{e}(t)+c_{0} e(t)=0(6)
$$

By examining the system in equation (6), it is easy to show that the solution of equation (6) ( or normal error) $\mathrm{e}(\mathrm{t})$ converges to zero with every positive gain $c_{0}$ as $\mathrm{t}$ goes to infinity, implying the state $\mathrm{x} 1$ converges to the command value $x_{r e f}$.

\section{INTEGRATOR-BACKSTEPPINGCONTROL}

The research starts with the concept of backstepping design for a MIMO strict-feedback model of nonlinear dynamic systems. A backstepping-based design is seen as a recursive design process which breaks a design problem on the full system down to a sequence of subproblems on lower order systems. Considering each lower order system with a Control Lyapunov Function (CLF) and paying attention to the interaction between two subsystems makes it modular and easy to design the stabilizing controller. A MIMO strict-feedback system in Eqs. (7) - (10) is addressed for formulating the stabilizing control law but a general form of nth order can be extended similarly.

Consider a MIMO strict-feedback form of nonlinear dynamic system in [16].

$\dot{x}_{1}=f_{1}\left(x_{1}, x_{2}, x_{3}\right)$

$\dot{x}_{2}=f_{2}\left(x_{1}, x_{2}, x_{3}, x_{4}\right)+g_{1}\left(x_{1}, x_{2}, x_{3}, x_{4}\right) u_{1}$

$\dot{x}_{3}=f_{3}\left(x_{1}, x_{2}, x_{3}, x_{4}\right)$

$\dot{x}_{4}=f_{4}\left(x_{1}, x_{2}, x_{3}, x_{4}\right)+g_{2}\left(x_{1}, x_{2}, x_{3}, x_{4}\right) u_{2}$ where $x \in \mathrm{R}^{4}, \mathrm{f}(\mathrm{x})$ and $\mathrm{g}(\mathrm{x})$ valued functions, $\mathrm{u} \in \mathrm{R}^{2}, \mathrm{y} \in \mathrm{R}^{2}, \mathrm{~h}(\mathrm{x})$ is -valuted function.

It is assumed that the system has a well-defined relative degree at equilibrium points and the $x_{2}, x_{4}$ variables in Eqs. (7) and (9) are solvable explicitly in terms of the other variables. Thus, input-output decoupling problem is solvable by a state space feedback exact linearization law and the state feedback law is designed using the backstepping approach.

The objective is to design a control law for the nonlinear control system (7)-(10) such that $x_{1} \rightarrow x_{1 \text { ref }}$, and $x_{3} \rightarrow$ $x_{3 \text { ref }}$ asymptotically where $x_{1 \text { ref }}$ and $x_{3 \text { ref }}$ are constant and globally asymptotical stability (GAS) in sense of the Lyapunov Theorem is achieved with no overshoots in the system. The following are the steps for formulating the IBSC laws for the system.

A backstepping control law for the strict-feedback model is formulated in principle in which the whole system is divided into $\mathrm{n}$ subsystems in a principle such that the ith subsystem consists of the (i-1)th subsystem plus an extra state and the nth subsystem is the original nth order system via coordinate transformations and feedbacks. By applying consecutively, the coordinate transformation and choosing a feedback law via the Control Lyapunov Function to each subsystem from the lowest to highest order and rewriting the feedback law in the original coordinates, the resulting controllers make the original deficient system a well-tracking command and asymptotically global stable. The following steps are used for formulating the IBSC for the system in Eqs. (7) - (10). 
Step 1: $x_{2}$ is regarded as a control input in Eq. (2) that is considered as the first subsystem. Thus, $x_{2}$ is chosen to make the first subsystem GAS. The choice is called avirtual control law, i.e.By putting

$$
\zeta_{1}=\left(x_{1}-x_{\text {ref }}\right)+c_{0} \sigma(11)
$$

Where $\sigma=\int_{0}^{t} e(\tau) d \tau, e(t)=\left(x_{1}-x_{\text {ref }}\right)$ and $e(t)$ is defined as normal tracking error and $c_{0}$ is a positive gain. By differentiating both sides of the Equation (8) in time and combining with the Equation (2), we have:

$\dot{\zeta}_{1}=\dot{x}_{1}=f_{1}\left(c_{0}, x_{\text {ref }}, \zeta_{1}+x_{1 r e f}, x_{2}, x_{3}\right)$

For the Equation (9), a CLF $V\left(\zeta_{1}\right)$ can be chosen such that when the virtual control law is applied, its time derivative becomes negative definite, i.e. $V_{1}=\frac{1}{2} \zeta_{1}^{2}$

By taking derivative in time of the CLF and combining with the Equation (9) results in;

$\dot{V}_{1}=\zeta_{1} \dot{\zeta}_{1}=\zeta_{1} f_{1}\left(c_{0}, x_{\text {ref }}, \zeta_{1}+x_{1 r e f}, x_{2}, x_{3}, \sigma\right)$

By satisfying the GAS condition in a sense of Lyapunov IBSC for the Equation (1), a virtual control law $\alpha_{1}$ can be chosen as follows;

$$
\begin{aligned}
-c_{1} \zeta_{1} & =f_{1}\left(c_{0}, x_{r e f}, \zeta_{1}+x_{1 r e f}, x_{2}, x_{3}, \sigma\right) \\
& \rightarrow \alpha_{1} \equiv x_{2}=\alpha_{1}\left(c_{0}, c_{1}, x_{r e f}, x_{1}, x_{3}, \sigma\right)(14)
\end{aligned}
$$

where $c_{1}$ is the positive gain. By doing so, we have:

$$
\dot{V}_{1}=\zeta_{1} \dot{\zeta}_{1}=-c_{1} \zeta_{1}^{2}<0 \forall \zeta_{1} \neq 0
$$

Step 2: By choosing the state feedback (14) and a change of coordinate (11) and (16), or

$$
\zeta_{2}=x_{2}-\alpha_{1}
$$

The second subsystem can be rewritten as follows:

$$
\left.\begin{array}{c}
\dot{\zeta}_{1}=-c_{1} \zeta_{1} \\
\dot{\zeta}_{2}=f_{2}\left(x_{1}, x_{2}, x_{3}\right)+g_{1}\left(x_{1}, x_{2}, x_{3}\right) u_{1}-\dot{\alpha}_{1}
\end{array}\right\}
$$

A CLF $V_{2}\left(\zeta_{1}, \zeta_{2}\right)$ can be chosen such that it makes the subsystem (17) GAS with the virtual control law, i.e.

$$
V_{2}=V_{1}+\frac{1}{2} \zeta_{2}^{2}
$$

Taking the derivative of the Equation (18) in time and combining with the Equation (17) result in;

$$
\begin{aligned}
\dot{V}_{2} & =-c_{1} \zeta_{1}^{2}+\zeta_{2}\left(f_{2}\left(\zeta_{1}+x_{1 r e f}, \zeta_{2}+\alpha_{1}, x_{3}, x_{4}\right)\right. \\
& \left.+g_{1}\left(\zeta_{1}+x_{1 r e f}, \zeta_{2}+\alpha_{1}, x_{3}, x_{4}\right) u_{1}-\dot{\alpha}_{1}\right)
\end{aligned}
$$

To meet the GAS condition in the sense of Lyapunov for the Equation (19), a control law $u_{1}$ can be chosen such that

$$
\begin{aligned}
-c_{2} \zeta_{2}= & f_{2}\left(\zeta_{1}, \zeta_{2}, x_{3}, x_{4}\right)+g_{1}\left(\zeta_{1}, \zeta_{2}, x_{3}, x_{4}\right) u_{1}-\dot{\alpha}_{1} \\
& \rightarrow u_{1}=\lambda_{2}\left(c_{0}, c_{1}, c_{2}, x_{r e f}, x_{1}, x_{2}, x_{3}, x_{4}, \sigma\right)(20)
\end{aligned}
$$

where $c_{2}$ is the positive gain. By doing so, we have:

$$
\dot{V}_{2}=-c_{1} \zeta_{1}^{2}-c_{2} \zeta_{2}^{2}<0 \forall \zeta_{1}, \zeta_{2} \neq 0
$$

Step 3: by choosing the state feedbacks (14) and (20) and a change of state transformations (11), (16), the third subsystem can be rewritten as follows:

$$
\left.\begin{array}{c}
\dot{\zeta}_{1}=-c_{1} \zeta_{1} \\
\dot{\zeta}_{2}=-c_{2} \zeta_{2} \\
\dot{x}_{3}=f_{3}\left(\zeta_{1}+x_{1 r e f}, \zeta_{2}+\alpha_{1}, x_{3}, x_{4}\right)
\end{array}\right\}
$$

By putting

$$
\zeta_{3}=\left(x_{3}-x_{3 r e f}\right)+c_{0} \sigma_{1}
$$

Where $\sigma_{1}=\int_{0}^{t} e_{1}(\tau) d \tau, e_{1}(t)=\left(x_{3}-x_{3 r e f}\right) \quad$ and $e_{1}(t)$ is defined as normal tracking error and $c_{0}$ is a positive gain. The third subsystem can be rewritten as follows:

$$
\left.\begin{array}{c}
\dot{\zeta}_{1}=-c_{1} \zeta_{1} \\
\dot{\zeta}_{2}=-c_{2} \zeta_{2} \\
\dot{\zeta}_{3}=f_{3}\left(c_{0}, c_{1}, x_{1 r e f}, x_{3 r e f}, x_{4}, \sigma_{1}\right)
\end{array}\right\}
$$

Now, $x_{4}$ is regarded as a control input in the subsystem (24). So, $x_{4}$ can be chosen to make the subsystem (24) GAS. A CLF $V_{3}\left(\zeta_{1}, \zeta_{2}, \zeta_{3}\right)$ can be chosen such that it makes the subsystem (24) GAS with the virtual control law, i.e.

$$
V_{3}=V_{2}+\frac{1}{2} \zeta_{3}^{2}
$$

By taking the derivative of the Equation (25) in time and combining with the Equation (24) result in;

$$
\dot{V}_{3}=-c_{1} \zeta_{1}^{2}-c_{2} \zeta_{2}^{2}+\zeta_{3} f_{3}\left(c_{0}, c_{1}, x_{1 r e f}, x_{3 r e f}, x_{4}, \sigma_{1}\right)
$$

To satisfy the GAS condition in the sense of Lyapunov for the Equation (26), a virtual control law $\alpha_{2}$ can be chosen such that

$$
\begin{gathered}
-c_{3} \zeta_{3}=f_{3}\left(c_{0}, c_{1}, x_{1 r e f}, x_{3 r e f}, x_{4}, \sigma_{1}\right) \\
\alpha_{3} \equiv \alpha_{3}\left(c_{0}, c_{1}, c_{2}, c_{3}, x_{1 r e f}, x_{3 r e f}, \zeta_{1}, \zeta_{2}, \xi_{3}, \sigma, \sigma_{1}\right)(27) \\
\text { where } c_{3} \text { is the positive gain. By doing so, we have: } \\
\dot{V}_{3}=-c_{1} \zeta_{1}^{2}-c_{2} \zeta_{2}^{2}-c_{3} \zeta_{3}^{2}<0 \forall \zeta_{1}, \zeta_{2}, \zeta_{3} \neq 0
\end{gathered}
$$

Step 4: by choosing the state feedbacks (14) (20) and (27) and a change of state transformations (11) (16) and (23), the complete system can be rewritten as follows:

$$
\left.\begin{array}{c}
\dot{\zeta}_{1}=-c_{1} \zeta_{1} \\
\dot{\zeta}_{2}=-c_{2} \zeta_{2} \\
\dot{\zeta}_{3}=-c_{3} \zeta_{3} \\
\dot{x}_{4}=f_{4}\left(\zeta_{1}+x_{1 r e f}, \zeta_{2}+\alpha_{1}, \zeta_{3}+x_{3 r e f}, x_{4}\right)+ \\
g_{2}\left(\zeta_{1}+x_{1 \text { ref }}, \zeta_{2}+\alpha_{1}, \zeta_{3}+x_{3 r e f}, x_{4}\right) u_{2}
\end{array}\right\}
$$

By putting

$$
\zeta_{4}=x_{4}-\alpha_{3}
$$

the complete system is rewrite as follows:

$$
\left.\begin{array}{c}
\dot{\zeta}_{1}=-c_{1} \zeta_{1} \\
\dot{\zeta}_{2}=-c_{2} \zeta_{2} \\
\dot{\zeta}_{3}=-c_{3} \zeta_{3} \\
\dot{\zeta}_{4}=f_{4}\left(\zeta_{1}+x_{1 \text { ref }}, \zeta_{2}+\alpha_{1}, \zeta_{3}+x_{3 r e f}, \zeta_{4}+\alpha_{3}\right) \\
+g_{2}\left(\zeta_{1}+x_{1 \text { ref }}, \zeta_{2}+\alpha_{1}, \zeta_{3}+x_{3 r e f}, \zeta_{4}+\alpha_{3}\right) u_{2}-\dot{\alpha}_{2}
\end{array}\right\}
$$

A CLF $V_{4}\left(\zeta_{1}, \zeta_{2}, \zeta_{3}, \zeta_{4}\right)$ can be chosen such that it makes the subsystem (31) GAS with control law, i.e.

$$
V_{4}=V_{3}+\frac{1}{2} \zeta_{4}^{2}
$$

By taking the derivative of the Equation (32) in time and combining with the Equation (31) Result in;

$\dot{V}_{4}=-c_{1} \zeta_{1}^{2}-c_{2} \zeta_{2}^{2}-c_{3} \zeta_{3}^{2}+\zeta_{4}\left(f_{4}+g_{2} u_{2}-\dot{\alpha}_{2}\right)(33)$ To satisfy the GAS condition in a sense of Lyapunov IBSC for the Equation (1), a control law $u_{2}$ can be chosen such that 


$$
\begin{gathered}
-c_{4} \zeta_{4}=f_{4}\left(\zeta_{1}, \zeta_{2}, \zeta_{3}, \zeta_{4}\right)+g_{1}\left(\zeta_{1}, \zeta_{2}, \zeta_{3}, \zeta_{4}\right) u_{2}-\dot{\alpha}_{2} \\
u_{2}=\alpha_{4}\left(c_{0}, c_{1}, c_{2}, c_{3}, c_{4}, x_{1 r e f}, x_{3 r e f}, x_{i}, \sigma, \sigma_{1}\right)(34)
\end{gathered}
$$

where $c_{4}$ is the positive gain. By doing so, we have:

$\dot{V}_{4}=-c_{1} \zeta_{1}^{2}-c_{2} \zeta_{2}^{2}-c_{3} \zeta_{3}^{2}-c_{4} \zeta_{4}^{2}<0 \forall \zeta_{I} \neq 0$

Thus, by choosing the state feedbacks (14) (20) (27) and (34) and a change of state transformations (11) (16), (23) and (30), the nonlinear control system (7) - (10) is transformed into the decoupled linear control system

$$
\left.\begin{array}{l}
\dot{\zeta}_{1}=-c_{1} \zeta_{1} \\
\dot{\zeta}_{2}=-c_{2} \zeta_{2} \\
\dot{\zeta}_{3}=-c_{3} \zeta_{3} \\
\dot{\zeta}_{4}=-c_{4} \zeta_{4}
\end{array}\right\}
$$

By examining the system (35), the system is stable and converges to zero with positive gains and the response of the system has no overshoots. The desired settling time and rising time of system are obtained by tuning the gains. Thus, the stability and performance specifications on the system (7)-(10) are achieved with the IBSC.In summary, the IBSC for the system (7) - (10) is given as follows

$$
\left.\begin{array}{c}
u_{1}=\alpha_{2}\left(c_{0}, c_{1}, c_{2}, c_{3}, x_{1 \text { ref }}, x_{3 r e f}, x_{i}, \sigma\right) \\
u_{2}=\alpha_{4}\left(c_{0}, c_{1}, c_{2}, c_{3}, c_{4}, x_{1 \text { ref }}, x_{3 r e f}, x_{i}, \sigma, \sigma_{1}\right)
\end{array}\right\}
$$

where $c_{0}, c_{1}, c_{2}, c_{3}$ and $c_{4}$ are positive gains that are determined optimally by using the modified genetic algorithm [13].

\section{APPLICATION OF NONLINEAR R2R WEB SYSTEM DYNAMICS}

Figure 1 shows two-span R2R web system that consists of unwinder, rewinder, infeeder, dancer system, two loadcells, rollers, and web lateral control system. The idle rollers guide the moving web around loadcell in a fixed angle. In order to control the web tension at span 1 and span 2, motors at unwinder and rewinder are used with control torques and respectively and the infeeder is used for the web velocity. The dancer system put at unwind side is to take up the slack during start-up and shutdown. On the other hand, two loadcells are used to feedback the web tension during the operating process and a web guide mechanism is used to control web lateral error. It is assumed that no web slippage occurs, the web has no permanent deformation due to applied tension, and the load cell and dancer dynamics is ignored.

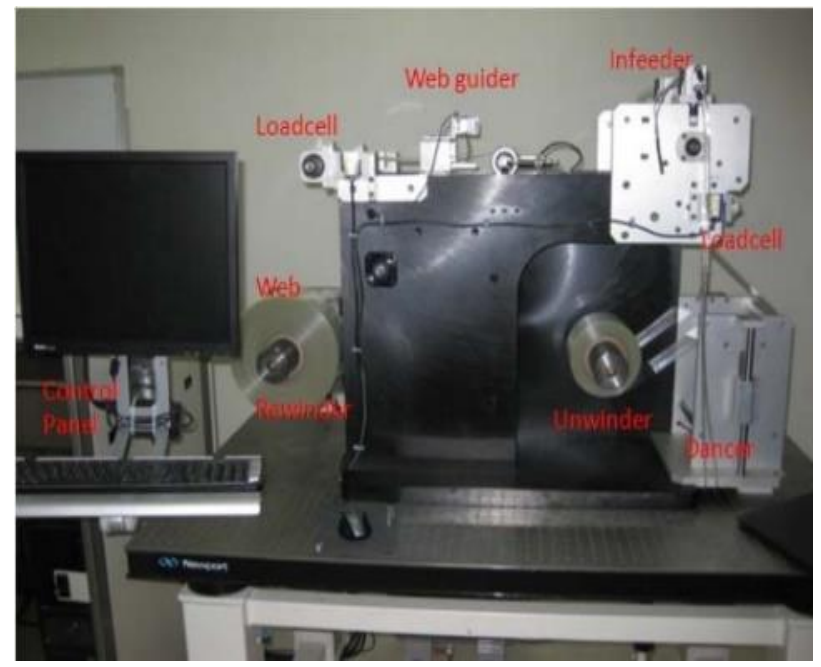

Figure 1. Two-Span Roll-to-RollWebSystem

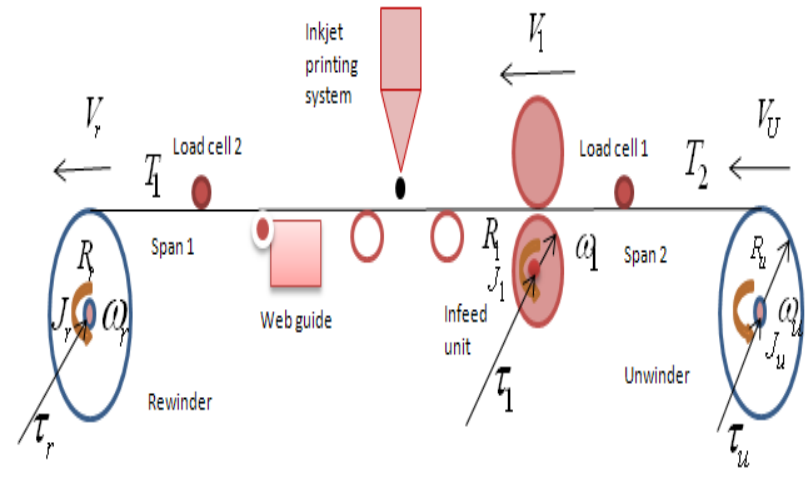

Figure 2 Model of Two-Span R2R Web System

By using Newton's law and the principle of mass conservation with assumptions, the non-linear dynamic equations of two-span roll-to-roll web control system can be written as follows.

$$
\begin{gathered}
\dot{\omega}_{1}=k_{7} T_{2}+k_{8} T_{1}+k_{9} \omega_{1}+k_{10} \tau_{1} \\
\dot{T}_{1}=k_{1} \omega_{1} T_{1}+k_{2} \omega_{1}+k_{3} \omega_{u} \\
\dot{\omega}_{u}=k_{4} T_{1}+k_{5} \omega_{u}+k_{6} \tau_{u} \\
\dot{T}_{2}=k_{11} \omega_{1} T_{1}+k_{12} \omega_{r} T_{2}+k_{13} \omega_{r}+k_{14} \omega_{1} \\
\dot{\omega}_{r}=k_{15} T_{2}+k_{16} \omega_{r}+k_{17} \tau_{r}
\end{gathered}
$$

Where $k_{1}=-\frac{R_{1}}{L_{1}}, k_{2}=\frac{E A R_{1}}{L_{1}}, k_{3}=-\frac{E A R_{u}}{L_{1}}, k_{4}=$ $\frac{R_{u}}{J_{u}}, k_{5}=-\frac{B_{u}}{J_{u}}, k_{6}=\frac{1}{J_{u}}, k_{7}=\frac{R_{1}}{J_{1}}, k_{8}=-\frac{R_{1}}{J_{1}}, k_{9}=$ $-\frac{B_{1}}{J_{1}}, k_{10}=\frac{1}{J_{1}}, k_{11}=\frac{R_{1}}{L_{2}}, k_{12}=-\frac{R_{r}}{L_{2}}, k_{13}=$ $\frac{E A R_{r}}{L_{2}}, k_{14}=-\frac{E A R_{1}}{L_{2}}, k_{15}=-\frac{R_{r}}{j_{r}}, k_{16}=-B_{r} / J_{r}, k_{17}=$ $1 / j_{r}$

$T_{1}=$ web tensions of span $1, T_{2}=$ web tensions of span2, $\omega_{u}=$ angular velocity of unwinder, $\omega_{1}=$ angular velocity of infeeder, $\omega_{r}=$ angular velocity of rewinder, $J_{u}=$ inertia moment of unwinder and motor at unwinder, $J_{1}=$ inertia moment of roller 1 and motor at roller $1, J_{r}=$ inertia moment of winder and motor at winder, $R_{u}=$ radius of unwinder, $R_{1}=$ radius of infeeder roller $1, R_{r}=$ radii of 
rewinder, $L_{1}=$ the length of span $1, L_{2}=$ the length of span 2, E: Young's module of web materials, A: Area of cross-section, $\tau_{u}=$ control torque generated by the motor at unwinder, $\tau_{1}=$ control torque generated by the motor at infeeder roller $1, \tau_{r}=$ control torque generated by the motor at rewinder, $\rho=$ the density of the web, $\mathrm{h}=$ the thickness of web.

\section{Problem Statement}

The given problem is to design a nonlinear control law for control inputs $\tau 1, \tau \mathrm{u}, \tau \mathrm{r}$ for the system (36) - (40) so that web tensions T1 tracks T1ref and T2 tracks T2ref and angular velocity $\omega 1$ tracks $\omega$ ref asymptotically with zero or acceptable small overshoot in the presence of disturbance.

\section{IBSC Law Formulation}

The integrator-backstepping-based control design approach for the MIMO strict-feedback system is used for formulating an IBSC law $\tau 1, \tau \mathrm{u}, \tau \mathrm{r}$ for the system (36) - (40). In this approach, the complete system (36) - (40) is divided into five subsystems: the first subsystem consists of Eq. (36), the second subsystem consists of Eq. (36) in new state variable and Eq. (37), the third subsystem consists of Eqs. (36), (37) in new state variables and Eq. (38), the fourth subsystem consists of Eqs. (36)-(38) in new state variables and Eq. (38), and the fifth subsystem (or complete system) consists of Eqs. (36)-(39) in new state variables and Eq. (40). Similar steps for IBSC law formulation for the system 36)-(40) in Section 2 are used for synthesizing the IBSC law $\tau 1, \tau \mathrm{u}, \tau \mathrm{r}$. In this paper, such a detailed procedure is not presented for the concise purpose. By doing so, the IBSC law for the system (36) - (40) is achieved. With the achieved above controllers, a similar block diagram, as shown in s [16], [17], provides a control strategy for numerical simulation of two-span R2R web system in the next section.

\section{Numerical Simulation Studies}

Simulation Conditions: Data for R2R web system, as shown in Fig. 1, in [15], [16] are used for simulation implementation with the optimal gains in [14]. Numerical studies are implemented in two different operating profiles to show advantages of the proposed method. In Case 1, single-step command for web tensions T1ref $=20(\mathrm{~N})$, T2ref $=15(\mathrm{~N})$ and desired angular velocity on feeder $\omega 1=0.5 \mathrm{rad} / \mathrm{s}$ is presented during 5 seconds without disturbance. In Case 2, same operating conditions as Case 1 is used for simulation during 30 seconds in the presence of varying radii of rewinder and unwinder in the second profile. Simulation studies center only on web tension control due to its importance in printed electronics technology. Thus, simulation results on web tension are shown and discussed as follows:

\section{Simulation Result}

Case 1: Single-Step Commands for 5 seconds

The blueline(or dash line) in

Fig.3showsthewebtensionof span 1 and the red line (or solid line) in Fig. 3 shows the web tension of span 2. The results indicate the web tensions have a well-tracking behavior with no overshoot and asymptotical stability. This result is exactly coincided to the ones in [17] in which no integrator is added to the control process. The reason for this is that the radii of rewinder and unwinder are assumed to be constant, this is only true for a short period of time with slow web velocity. Thus, the numerical simulation is implemented for a long period of time as shown in Case 2.

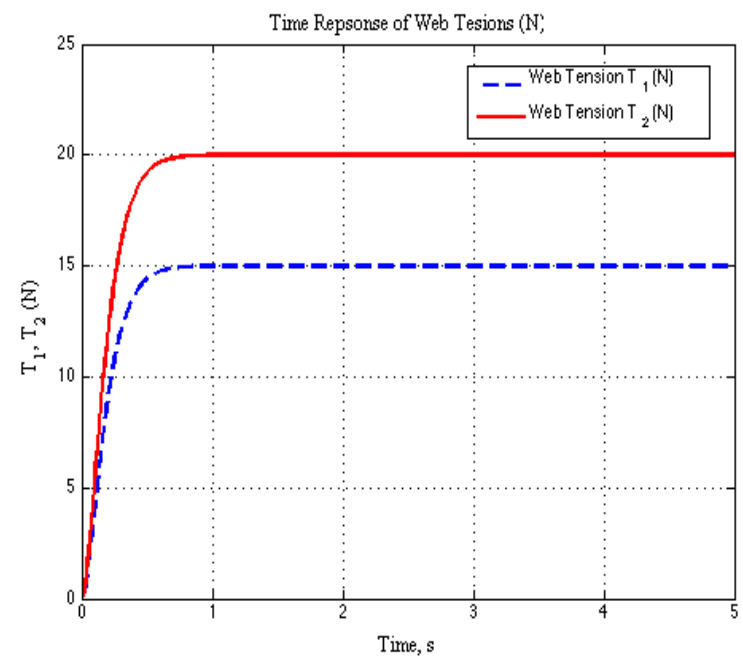

Figure 3 Time Response of Web Tensions of 5 seconds

Case 2: Single-Step Commands for 30 seconds

The blue line (or dash line) in Fig. 4 shows the web tension of span 1 without an integrator and the red line (or solid line) in Fig. 4 shows the web tension of span 2 without an integrator. The results indicate the web tensions have a divergence as time increases. Particularly, the web tension in span 1 increases as the radius of rewinder increases and the web tension in span 2 decreases as the radius of unwinder decreases. Similarly, the green line (or solid line) in Fig. 4 shows the web tension of span 1 with an integrator and the black line (or dot line) in Fig. 4 shows the web tension of span 2 with an integrator. Results show that the web tensions track asymptotically to the commands with stability as two integrators are used. This verifies the theoretical prediction, as shown in Theorem 2. 


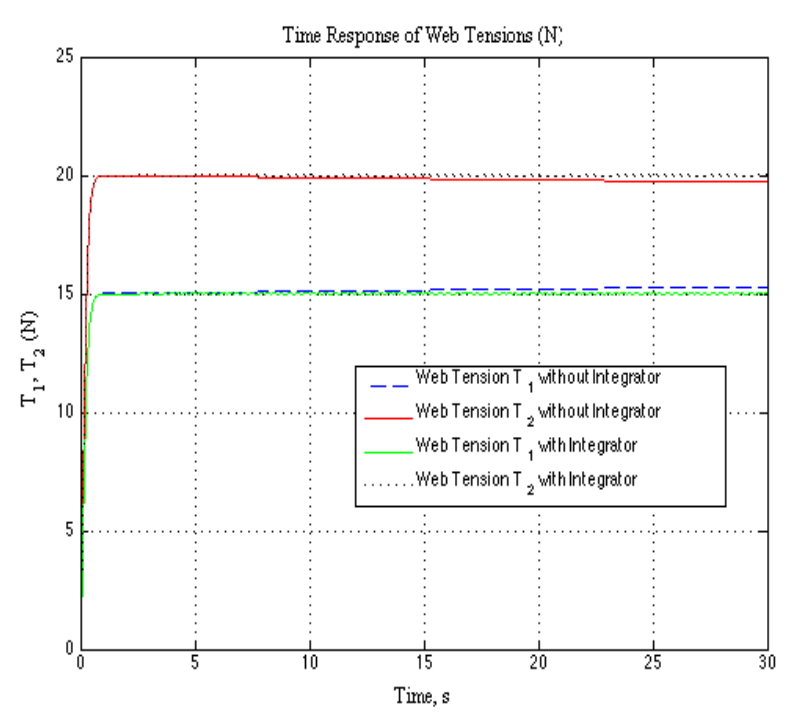

Figure 4 Time Response of Web Tensions of 30 seconds

\section{CONCLUSION}

In this paper, an IBSC-based control method is provided for a general MIMO strict-feedback model of nonlinear dynamic systems and then is applied for R2R web dynamic system. The IBSC-based control method can adapt and eliminate the varying parameters of R2R web system and provides with a robust control approach for varying-time parameter systems. Observing the simulation results and the above discussion, conclusions are made as follows: A systematic procedure is presented for formulating the IBSC law for a MIMO strict-feedback model; Application for R2R web system indicates that the IBSC-based control design achieves the performance specifications and stability; The IBSCbased control method provides a robust control strategy with a high accuracy in the presence of disturbance. With the rapid development of sensors and electronic devices, the IBSC-based control algorithm can result in a control system with high precision and are useful for applications with high digital computational system.

\section{ACKNOWLEDGMENTS}

This is supported by the Ministry of Education and Training (MOET) Funded Project of Code KC-519.

\section{REFERENCES}

[1] P. V. Kokotovc, "The joy of feedback: Nonlinear and adaptive," IEEE, 1992.

[2] T. T. Tran, Nonlinear Flight Control Design Using Backstepping Methodology, Dissertation, 16, Department of Mechanical and Aerospace Engineering, Old Dominion University, 2016.

[3] K. C. Lin, M. C. Tsai, and K. Y. Chen, "Web tension control of a start-up process using observer techniques with friction and inertia compensation," The 27th annual conference of the IEEE industrial electronics society, pp. 23-28, 2001.

[4] K. C. Lin, "Observer-based tension feedback control with friction and inertia compensation," IEEE Transactions on control systems technology, Vol. 11, No 1, pp. 109-118, 2003.

[5] C. G. Kang and B. S. Lee, "Stability analysis for design parameters of a roll-to-roll printing machine," International Conference on Control, Automation and Systems 2007, pp. 23 - 44, 2007.

[6] S. H. Song and S. K. Sul, "A new tension controller for continuous strip processing line," IEEE Transactions on control systems technology, Vol. 11, No 1, pp. 2225-2230, 1998.

[7] S. R. Oh, "The design of web tension control system using a nonlinear feedback controller," KIEE, Vol. 55D, No 1, pp. 14-16, 2006.

[8] D. Knittel, E. Laroche, and H. Koc, "Tension control for winding systems with two degrees of freedom $\mathrm{H}$ ? controller," IEEE Transactions on control systems technology, Vol. 11, No 1, pp. 576-582, 2001.

[9] A. F. Lynch, S. A. Bortoff, and K. Robenack, "Nonlinear tension observer for web machine," Automatica, No. 40, pp.1517-1524, 2004.

[10] T. Sakamoto and Y. Izumihara, "Decentralized control strategies for web tension control system," IEEE Transactions on control systems technology, No. 97TH8280, pp. 1086-1089.

[11] K. H. Shin, J. I. Jang, H. K. Kang, and S. H. Song, "Compensation method for tension disturbance due to an unknown roll shape in a web transport system," IEEE Transactions on Industry and Applications, Vol. 39, No 5, 2003.

[12] S. P. Nagarkatti, F. Zhang, and C. D. Rahn, and D. M. Dawson, "Tension and speed regulation for axially moving materials," Journal of Dynamic Systems, Measurement, and Control, Vol. 122, pp.445-436, 2003.

[13] T. Sakamoto and Y. Fujino, "Modeling and analysis of a web tension control system," IEEE Catalog Number: 95TH8081, pp. 358-362, 1995.

[14] K. H. Choi, T. T. Tran, and D. S. Kim, "A new approach for designing the controller of single-span roll-to-roll web system using the modified backstepping technique," International Journal of Intelligent Systems Technologies and Applications, Vol. 9, No. 3/4, 2010.

[15] K. H. Choi, T. T. Tran, and D. S. Kim, "Backstepping Controller Based Web Tension Control for Roll-to-Roll Web Printed Electronics System," Journal of Advanced Mechanical Design, Systems, and Manufacturing, Vol. 5, No. 1, pp. 7-21, 2011.

[16] T. T. Tran, K. H. Choi, D. E. Chang and D. S. Kim, "Web Tension and Velocity Control of Two-span 
Roll-to-roll System for Printed Electronic,” Journal of Advanced Mechanical Design, Systems, and Manufacturing, Vol. 5, No. 4, pp. 329-346, 2011.

[17] T. T. Tran and K. H. Choi, "A Backstepping Based Control Algorithm for Multi-Span Roll-to-roll Web System," International Journal ofAdvanced Manufacturing Technology, Vol. 70, No. 1, pp. 45$61,2013$.
[18] T. T. Tran and B. Newman, "IntegratorBackstepping Control Design for Nonlinear Flight System Dynamics," AIAA Conference on Guidance, Navigation, and Control Conference, AIAA 20151321, 2015. 